\title{
Carboxyhaemoglobin levels in water-pipe and cigarette smokers
}

\author{
Ansa Theron, Cedric Schultz, James A Ker, Nadia Falzone
}

Water-pipe smoking is growing in popularity, especially among young people, because of the social nature of the smoking session and the assumption that the effects are less harmful than those of cigarette smoking. It has however been shown that a single water-pipe smoking session produces a 24-hour urinary cotinine level equivalent to smoking 10 cigarettes per day.

Aim. We aimed to measure carboxyhaemoglobin $(\mathrm{COHb})$ blood levels before and after water-pipe and cigarette smoking sessions.

Method. Self-confessed smokers older than 18 years $(N=30)$ volunteered to smoke a water-pipe or a cigarette and have their blood $\mathrm{COHb}$ levels measured under controlled conditions.

Results. Mean baseline $\mathrm{COHb}$ levels were $2.9 \%$ for the 15 cigarette smokers and $1.0 \%$ for the 15 water-pipe smokers. Levels increased by a mean of $481.7 \%$ in water-pipe smokers as opposed to $39.9 \%$ in cigarette smokers.

Conclusion. The study demonstrated that water-pipe smokers had significantly higher increases in blood $\mathrm{COHb}$ levels than cigarette smokers during a single smoking session.

S Afr Med J 2010; 100: 122-124.
Water-pipes (hookah, narghile, shisha, goza, hubble bubble and in South Africa hubbly-bubbly) have been used to smoke tobacco for more than 400 years. Traditionally in the Middle East water-pipe smoking has been the habit of older men, but it is increasing in popularity worldwide, especially among students, other young people and children. ${ }^{1}$ The popularity of water-pipe smoking appears to be based on its social nature and the assumption that its effects are less harmful than those of cigarette smoking. ${ }^{2}$ The increase in water-pipe smoking can partly be attributed to the popularity of the sweetened and variously flavoured tobaccos that are used in the water-pipe.

A water-pipe is essentially a glass bottle with hoses and a mouthpiece through which to smoke. ${ }^{3}$ The head contains the tobacco, and since the tobacco is too moist to burn on its own, charcoal is placed on top of the tobacco-filled head. The body is fixed to the neck of the bottle, and its central conduit is submerged in the bottle. The bottle is half-filled with water and placed on the ground. The hose is attached to an aperture in the side of the pipe (Fig. 1). When the charcoal is lit, the smoker inhales through the hose. This creates a vacuum above the water, drawing air through the body and over the tobacco

Lung Unit, Department of Internal Medicine, Steve Biko Academic Hospital, and School of Medicine, University of Pretoria

Ansa Theron, BTech

Cedric Schultz, DTech

James A Ker, MB ChB, MMed, MD

Tshwane University of Technology, Pretoria

Nadia Falzone, PhD (currently at Oxford University)

Corresponding author: A Theron (theronansa@gmail.com) and charcoal. The smoke bubbles through the water, where it is diluted and cooled, and then inhaled by the smoker.

Carbon monoxide (CO) is produced by the incomplete combustion of carbon-containing material, notably tobacco, biomass fuel and fossil fuel. The carboxyhaemoglobin $(\mathrm{COHb})$ level in the blood (half-life 2 - 6 hours) after inhalation of $\mathrm{CO}$ will depend on concentrations of inspired $\mathrm{CO}$, duration of exposure, pulmonary ventilation and the $\mathrm{COHb}$ level before the inhalation. ${ }^{4} \mathrm{COHb}$ is a sensitive specific physiological marker of atmospheric $\mathrm{CO}$ exposure from both indoor and outdoor sources.

In water-pipe smokers expired $\mathrm{CO}$ was reported to increase by $300 \%$ after an hour of smoking, while in cigarette smokers it only increased by $60 \% .^{5}$

During a single water-pipe smoking session a smoker may produce a 24-hour urinary cotinine level that is equivalent to smoking 10 cigarettes a day $(95 \%$ confidence interval (CI) 7 - 13 cigarettes a day). ${ }^{6}$ Contrary to popular belief, noxious substances such as nicotine, tar and heavy metals (chromium, arsenic, lead) are found in the smoke of water-pipes. ${ }^{7}$ Although water-pipe smoking is so widespread, its adverse health effects are not well documented. Data on CO levels in the blood after a controlled smoking session comparing water-pipe smoking with cigarette smoking are limited.

We aimed to measure $\mathrm{COHb}$ levels in the blood before and after water-pipe smoking and cigarette smoking under controlled conditions.

\section{Materials and methods}

Only self-confessed smokers of cigarettes and water-pipes aged 18 years and older were recruited from students and hospital workers. There were no regular pipe smokers among 


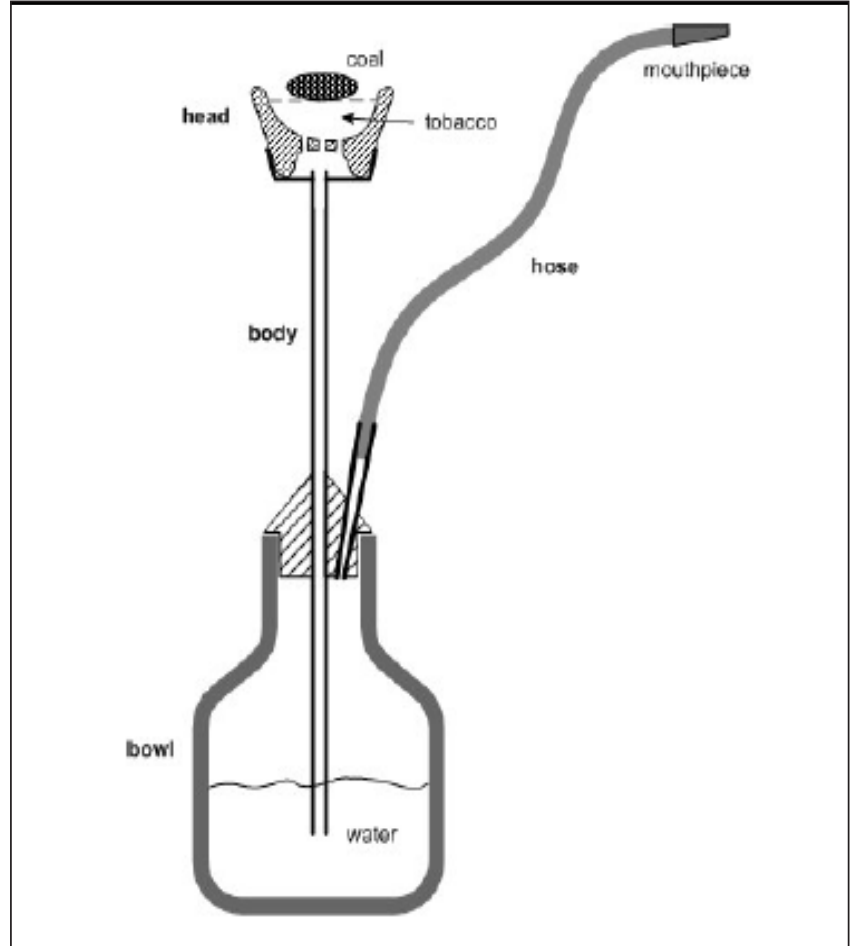

Fig. 1. A cross-section of the components of a water-pipe.

the participants. The subjects were required to smoke either a cigarette or a water-pipe. A venous blood sample was drawn before and after smoke exposure. The number of inhalations during the smoking session and the duration of the exposure were noted. The $\mathrm{COHb}$ levels in the pre- and post-exposure blood samples were measured.

There were 15 participants in each group (cigarette smokers and water-pipe smokers). The subjects were grouped according to smoking preference. Persons with a history of pulmonary disease and pregnant women were excluded.

The blood samples were drawn using a sterile venepuncture kit and analysed in a Radiometer ABL 800 Flex (Denmark) blood gas apparatus, calibrated and maintained according to international standards of the American Association for Respiratory Care (AARC). ${ }^{8}$

The cigarette and water-pipe smoking session took place in a designated smoking area at the hospital. After the procedure had been explained to the participants and they had signed informed consent, pre-exposure blood samples were drawn and analysed. The participants then smoked as per preference. After the smoking session, post-exposure blood samples were drawn and analysed within 5 minutes of smoking cessation. $\mathrm{COHb}$ levels before and after smoking were measured and the number of inhalations and the length of smoking exposure documented. The percentage change between levels before and after smoking was calculated using the formula: postmeasurement - pre-measurement/pre-measurement $\times 100$.

\section{Data analysis}

All descriptive analyses of means, standard deviations (SDs), 95\% CIs and correlations were assessed. A paired-sample $t$-test and an independent two-sample $t$-test were used for analysis of the pre- and post-COHb levels of the two groups of smokers. This method of analysis was selected because the participants were not randomised. Correlations were analysed using the Spearman rank test. All statistical analyses were done using statistics software Statistix 3.0.

\section{Ethics}

The study was approved by the Student's Ethics Committee of the Tshwane University of Technology and the Pretoria University Ethics Committee. All participants gave written consent. To protect privacy, the study data were encoded.

\section{Results}

Fifteen cigarette smokers ( 9 male, 6 female) and 15 water-pipe smokers (10 male, 5 female) were entered in the respective groups. The mean age of the cigarette smokers was 32 years compared with 22.3 years for the water-pipe smokers $(p<0.05)$.

The means and SDs for the pre- and post-exposure $\mathrm{COHb}$ levels, the percentage change between the pre- and postexposure levels, the number of inhalations and the duration of the smoking session are set out in Table I. The mean baseline $\mathrm{COHb}$ level for the cigarette smokers was 2.9\% (SD 1.4) and that for the water-pipe smokers $1.0 \%$ (SD 0.4) $(p<0.02)$.

There were statistically significant differences between the pre-smoking and post-smoking $\mathrm{COHb}$ levels, the percentage change in levels, the number of inhalations, the duration of exposure and the age of the participants in the water-pipe

Table I. Demographic data and smoking data for the 30 study participants

\begin{tabular}{|c|c|c|c|c|c|c|}
\hline & $\begin{array}{l}\text { Pre-exposure } \\
\mathrm{COHb}\end{array}$ & $\begin{array}{l}\text { Post-exposure } \\
\mathrm{COHb}\end{array}$ & $\begin{array}{l}\text { \% change } \\
\text { (SD) }\end{array}$ & $\begin{array}{l}\text { No. of } \\
\text { inhalations } \\
\text { (SD) }\end{array}$ & $\begin{array}{l}\text { Time (min) } \\
\text { (SD) }\end{array}$ & $\begin{array}{l}\text { Age (yrs) } \\
\text { (SD) }\end{array}$ \\
\hline Cigarette smokers & $2.9(1.4)$ & $3.7(1.4)$ & $39.9(55.1)$ & $11.5(4.2)$ & $6.3(1.7)$ & 32 \\
\hline $\begin{array}{l}\text { Water-pipe } \\
\text { smokers }\end{array}$ & $1.0(0.4)$ & $5.8(3.7)$ & $481.7(330.1)$ & $45.3(38.3)$ & $24.9(16.0)$ & 22.3 \\
\hline$p$-value & $<0.0001$ & $<0.001$ & $<0.001$ & $<0.001$ & $<0.0001$ & $<0.05$ \\
\hline
\end{tabular}




\section{Original Articles}

smokers compared with the cigarette smokers. Repeated measure analysis of variance was done to adjust for the higher baseline $\mathrm{COHb}$ and age of the cigarette smoking group $(p<0.0001)$.

Increases in $\mathrm{COHb}$ were highest among the participants who took the most inhalations and/or had the longest exposure. The mean pre- and post-exposure $\mathrm{COHb}$ levels of cigarette smokers and water-pipe smokers, and the mean percentage change between the pre- and post-exposure levels in the two groups, are set out in Table I. The percentage change in $\mathrm{COHb}$ levels was much larger in the water-pipe group (mean 481.7\%) than in the cigarette group (mean 39.9\%).

The correlation (Spearman) between the number of inhalations and the post-exposure $\mathrm{COHb}$ levels was $r=-0.28$ for the cigarette smokers and $r=0.78$ for the water-pipe smokers. The correlations between the durations of exposure and the post-exposure $\mathrm{COHb}$ levels in the cigarette smokers and the water-pipe smokers were $r=0.28$ (no correlation) and $r=0.93$ (strong correlation), respectively.

\section{Discussion}

The gender distribution was approximately the same for the two groups in this study, but the cigarette smokers were significantly older than the water-pipe smokers. This may reflect the increasing popularity of water-pipe smoking among young people globally, but bias could have played a role in selecting the participants.

The baseline $\mathrm{COHb}$ level of the cigarette smokers was higher than that of the water-pipe smokers, possibly because water-pipe smoking typically occurs over weekends as a social activity, while cigarette smoking is a daily habit.

The strong correlation between post-exposure $\mathrm{COHb}$ and both the number of inhalations and the length of exposure in water-pipe smoking could be because the water-pipe smoke is cooled as it passes through the water and is much less irritating to the airways than cigarette smoke, enabling the smoker to smoke more and longer. It could also be related to the fact that water-pipe smoking may be associated with larger side-stream smoke volumes ('second-hand smoke'), increasing exposure to smoke over time. Three participants in the water-pipe group had smoking sessions similar to those in the cigarette group with regard to number of inhalations and length of exposure, and still showed an increase in $\mathrm{COHb}$ greater than $120 \%$ compared with a mean of $39 \%$ in the cigarette group.

Other factors that could have affected the results are depth of inhalation and second-hand smoke. It is possible that differences in smoking techniques such as depth of inhalation could have influenced the results in both groups, along with physiological factors such as the diffusion of gases across the respiratory membranes and transport of gases in the blood. Water-pipe smoking also appears to produce a lot of smoke, and as it is generally done in groups, it is possible that the participants have more second-hand smoke exposure than cigarette smokers do. A study of habitual water-pipe smokers in Saudi Arabia found them to have a mean $\mathrm{COHb}$ level of $10.06 \%$ (SD 2.50\%) compared with $1.60 \%$ (SD $0.20 \%$ ) for nonsmokers and $6.47 \%$ (SD 2.73\%) for cigarette smokers. ${ }^{9}$ In contrast, our study demonstrated an acute rise in $\mathrm{COHb}$ levels in a single smoking session under controlled conditions.

In conclusion, this study demonstrated that water-pipe smokers had significantly higher increases in $\mathrm{COHb}$ levels than cigarette smokers in a single smoking session under controlled conditions. The rise in $\mathrm{COHb}$ levels indicates that smoking a water-pipe is not harmless and may have deleterious longterm health effects. Young people should be warned about the possible hazards of water-pipe smoking.

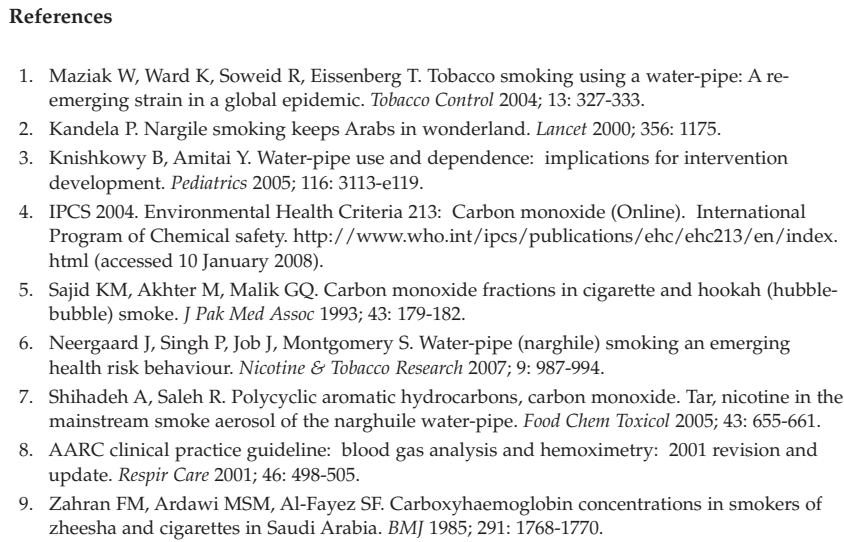

Accepted 10 July 2009. 